\title{
GENERAL SADDLEPOINT APPROXIMATIONS: APPLICATION TO THE ANDERSON-DARLING TEST STATISTIC
}

\author{
Qian Chen \\ China Academy of Public Finance and Public Policy, \\ Central University of Finance and Economics, \\ Beijing, People's Republic of China \\ \& \\ David E. Giles \\ Department of Economics, University of Victoria, \\ Victoria, B.C., Canada
}

May 2007

\begin{abstract}
We consider the relative merits of various saddlepoint approximations for the c.d.f. of a statistic with a possibly non-normal limit distribution. In addition to the usual Lugannani-Rice approximation we also consider approximations based on higher-order expansions, including the case where the base distribution for the approximation is taken to be non-normal. This extends earlier work by Wood et al. (1993). These approximations are applied to the distribution of the Anderson-Darling test statistic. While these generalizations perform well in the middle of the distribution's support, a conventional normal-based Lugannani-Rice approximation (Giles, 2001) is superior for conventional critical regions.
\end{abstract}

Keywords: Saddlepoint approximation; Goodness-of-fit; Anderson-Darling test

Mathematics Subject Classification: Primary 62E17, 62G10; Secondary 62G30

JEL Classifications: C13, C22, F31, F41

Proposed Running Head: SADDLEPOINT APPROXIMATIONS

\section{Author Contact:}

David Giles, Dept. of Economics, University of Victoria, P.O. Box 1700, STN CSC, Victoria, B.C., Canada V8W 2Y2; e-mail: dgiles@uvic.ca; FAX: (250) 721-6214 


\section{GENERAL SADDLEPOINT APPROXIMATIONS: APPLICATION TO THE ANDERSON-DARLING TEST STATISTIC}

\section{INTRODUCTION}

The problem of approximating the density or distribution function of a statistic is of great practical importance. Saddlepoint approximations can be obtained for any statistic that admits a cumulant generating function. In principle, knowledge of the cumulant generating and characteristic functions allows us to obtain the density and distribution functions using the inversion theorem. However, in practice the complexity of the associated integration may make it very costly (or impossible) to obtain an exact analytic result. Compared with other asymptotic approximations, saddlepoint approximations (Daniels, 1954) have the advantages of always generating positive probabilities; being very accurate in the tails of the distribution; and being accurate with small samples, sometimes even with only one observation.

Saddlepoint approximations have proved useful for a range of problems, and hence have attracted considerable interest. With an appropriate renormalization to ensure that the saddlepoint density function integrates to unity over its support, the accuracy of the approximation can sometimes be improved, and in certain cases it even reproduces the exact density. Daniels (1980) showed that the normal, gamma and inverse normal are the only cases for which the renormalized saddlepoint approximation reproduces the exact density in the scalar case. This also occurs for normal and inverse normal densities without renormalization. 
Lugannani and Rice (1980) proposed a saddlepoint approximation for the cumulative distributive function that is very accurate in the tails. Of course, this is of great interest in testing problems. Other approaches for approximating the tail areas are discussed by Barndorff-Nielsen (1991), and Daniels (1987) provides a full discussion of this issue. Routledge and Tsao (1997) prove that the derivation of Lugannani and Rice's asymptotic expansion for the cumulative distribution function is the same as Daniels's asymptotic approximation for the corresponding density function. A comprehensive review of the application of saddlepoint approximations can be found in Reid $(1988,1991)$ and Field and Ronchetti (1990), and useful introductory discussions are provided by Goustis and Casella (1999) and Huzurbazar (1999).

All of the approximations noted above use a normal base distribution, and are generally accurate when the statistic has a normal limiting distribution. Wood, et al. (1993) proposed a generalization involving a non-normal-based saddlepoint approximation and showed that it can be more accurate than the normal-based approximation in some important cases when the limit distribution is non-normal. In this paper we consider four saddlepoint approximations - those of Lugannani and Rice and Wood et al., together with their counterparts that include higher order terms in the underlying expansions. The higher-order variant of Wood et al.'s non-normal-based approximation appears not to have been considered previously. These approximations are compared by applying them to the distribution of the widely used Anderson and Darling $(1952,1954)$ goodness-of-fit test statistic. 
The structure of this paper is as follows. In section 2, we present some saddlepoint theory. In section 3 we discuss the Anderson-Darling (A-D) test and some of its properties, and derive the various saddlepoint approximations for the distribution of the A-D statistic. We compare some numerical evaluations of these saddlepoint approximations with Lewis's (1961) numerical results in section 4. Concluding comments follow in section 5 .

\section{SADDLEPOINT APPROXIMATIONS}

\subsection{Background}

The Lugannani-Rice (L-R) (normal-based) approximation for the distribution of the random variable $X$ at point $y$ is:

$$
\operatorname{Pr}(X \geq y) \approx 1-\Phi(\hat{w})+\phi(\hat{w})\left\{\frac{1}{\hat{u}}-\frac{1}{\hat{w}}\right\}
$$

where $\Phi$ and $\phi$ are the c.d.f. and p.d.f of the standard normal distribution, and

$$
\begin{aligned}
& \hat{w}=\{2[\hat{t} y-K(\hat{t})]\}^{1 / 2} \operatorname{sgn}(\hat{t}) \\
& \hat{u}=\hat{t}\left[K^{(2)}(\hat{t})\right]^{1 / 2} .
\end{aligned}
$$

Here, $K(t)$ is the cumulant generating function (c.g.f.) of the variable $X, K^{(i)}(\cdot)$ is its $i^{\text {th }}$ derivative, and $\hat{t}$ is the solution to the saddlepoint equation:

$$
K^{(1)}(\hat{t})=y \quad .
$$

where

$$
\operatorname{sgn}(\hat{t})= \begin{cases}+1, & \text { if } \hat{t}>0 \\ -1, & \text { if } \hat{t}<0 \\ 0, & \text { if } \hat{t}=0\end{cases}
$$


When $y=E(X)=K^{(1)}(0), \hat{u}$ and $\hat{w}$ are zero, and (1) collapses. Applying L'Hôpital's rule in this case, (1) reduces to:

$$
\operatorname{Pr}(X \geq E(x)) \approx \frac{1}{2}-\frac{\zeta_{3}(0)}{6 \sqrt{2 \pi}}
$$

where $\zeta_{r}(\hat{t})=K^{(r)}(\hat{t}) /\left\{K^{(2)}(\hat{t})\right\}^{r / 2}$.

The existence of a unique real root to the saddlepoint equation is established by Daniels (1954).

\section{$2.2 \quad$ Non-normal-based saddlepoint approximation}

Wood et al. (1993) generalize the L-R formula to a non-normal-based (WBB) saddlepoint approximation, by first making the transformation:

$$
G\left(w_{\hat{\varepsilon}}\right)-\hat{\varepsilon} w_{\hat{\varepsilon}}=K(\hat{t})-y \hat{t}
$$

where $\hat{t}$ is obtained from equation (4), $w_{\widehat{\varepsilon}}$ is the solution to the saddlepoint equation:

$$
G^{(1)}\left(w_{\hat{\varepsilon}}\right)=\hat{\varepsilon},
$$

and $G^{(r)}(\cdot)$ denotes the $r^{\text {th }}$ derivative of $G(\cdot)$.

The WBB formula is:

$$
\operatorname{Pr}(X \geq y) \approx 1-\Gamma(\hat{\varepsilon})+\gamma(\hat{\varepsilon})\left\{\frac{1}{\hat{u}_{\varepsilon}}-\frac{1}{w_{\hat{\varepsilon}}}\right\},
$$

where $\Gamma$ and $\gamma$ are the c.d.f. and p.d.f. of the base distribution whose c.g.f. is $G$. $\hat{\varepsilon}$ and $w_{\hat{\varepsilon}}$ are defined by (6) and (7). Clearly, we need to solve the root $\hat{t}$ before we calculate $\hat{\varepsilon}$ 
and $w_{\hat{\varepsilon}}$. After finding $\hat{t}$, we can express $w_{\hat{\varepsilon}}$ as a function of $\hat{\varepsilon}$ based on (7), then we substitute this function into (6) to solve for $\hat{\varepsilon}$ numerically. Finally, we can solve for $w_{\hat{\varepsilon}}$. The left-hand side of (6) is the Legendre-Frenchel transformation of $G$., which is concave in $\hat{\varepsilon}$. Therefore there are at most two solutions for $\hat{\varepsilon}$, and the choice for the solution of $\hat{\varepsilon}$ is:

$$
\hat{\varepsilon}= \begin{cases}\varepsilon_{+}(y), & \text { if } y>K^{(1)}(0) \\ \varepsilon_{-}(y), & \text { if } y<K^{(1)}(0) \\ G^{\prime}(0), & \text { if } y=K^{(1)}(0)\end{cases}
$$

and

$$
\hat{u}_{\hat{\varepsilon}}=\hat{u}\left[G^{(2)}\left(w_{\hat{\varepsilon}}\right)\right]^{-1 / 2}
$$

where $\hat{u}$ is defined by (3).

When $y=E(X)=K^{(1)}(0), \hat{\varepsilon}=G^{(1)}(0)$ from (9). Then from (7), $w_{\hat{\varepsilon}}=0$ and $\hat{u}_{\hat{\varepsilon}}=0$, for the same reason as with the L-R formula. Therefore, at the mean value, we should calculate the limit of (8), which is:

$\operatorname{Pr}(X \geq E(x)) \approx 1-\Gamma\left(G^{(1)}(0)\right)+\frac{1}{6} \sqrt{G^{(2)}(0)} \gamma\left(G^{(1)}(0)\right)\left\{\zeta_{3}^{\prime}(0)-\zeta_{3}(0)\right\}$

where $\zeta_{r}^{\prime}\left(w_{\hat{\varepsilon}}\right)=G^{(r)}\left(w_{\hat{\varepsilon}}\right) /\left\{G^{(2)}\left(w_{\hat{\varepsilon}}\right)\right\}^{r / 2}$.

\subsection{Higher-order saddlepoint approximations}

Daniels (1987, p.42) extended the Lugannani-Rice formula to incorporate higher-order terms for the mean of $n$ independent identically distributed random variables: 


$$
P(\bar{X}>\bar{x}) \approx 1-\Phi\left(\hat{w} n^{1 / 2}\right)+\phi\left(\hat{w} n^{1 / 2}\right)\left\{\frac{b_{0}}{n^{1 / 2}}+\frac{b_{1}}{n^{3 / 2}}+\ldots+\frac{b_{k}}{n^{(k+1) / 2}}+o\left(n^{-k-3 / 2}\right)\right\}
$$

where

$$
b_{0}=\frac{1}{\hat{u}}-\frac{1}{\hat{w}}, \quad b_{1}=\frac{1}{\hat{u}}\left(\frac{\zeta_{4}}{8}-\frac{5}{24} \zeta_{3}^{2}\right)-\frac{\zeta_{3}}{2 \hat{u}^{2}}-\frac{1}{\hat{u}^{3}}+\frac{1}{\hat{w}^{3}}
$$

and for brevity, we write $K^{(r)}(\hat{t}), G^{(r)}\left(w_{\hat{\varepsilon}}\right), \zeta_{(r)}(\hat{t})$ and $\zeta_{(r)}^{\prime}\left(w_{\hat{\varepsilon}}\right)$ as $K^{(r)}, G^{(r)}, \zeta_{(r)}$ and $\zeta_{(r)}^{\prime}$. For a single random variable, $X$, Daniels's result becomes:

$$
P(X>y) \approx 1-\Phi(\hat{w})+\phi(\hat{w})\left\{b_{0}+b_{1}+\ldots+b_{k}+\ldots\right\} .
$$

To get a higher-order approximation for the c.d.f. we retain both $b_{0}$ and $b_{1}$ terms in (14):

$\operatorname{Pr}(X \geq y) \approx 1-\Phi(\hat{w})+\phi(\hat{w})\left\{\left(\frac{1}{\hat{u}}-\frac{1}{\hat{w}}\right)+\frac{1}{\hat{u}}\left(\frac{\zeta_{4}}{8}-\frac{5}{24} \zeta_{3}^{2}\right)-\frac{\zeta_{3}}{2 \hat{u}^{2}}-\frac{1}{\hat{u}^{3}}+\frac{1}{\hat{w}^{3}}\right\}$.

At the mean, (15) reduces to

$\operatorname{Pr}(X \geq E(X)) \approx \frac{1}{2}-\frac{1}{2 \pi}\left\{\frac{1}{6} \zeta_{3}-\frac{1}{40} \zeta_{5}+\frac{5}{48} \zeta_{3} \zeta_{4}-\frac{35}{432} \zeta_{3}^{2}\right\}$.

A higher-order WBB formula can also be obtained in an analogous manner, the details of which appear in the Appendix:

$$
\begin{aligned}
\operatorname{Pr}(X \geq y) \approx 1 & -\Gamma(\hat{\varepsilon})+\frac{\gamma(\hat{\varepsilon})}{\delta_{\hat{\varepsilon}}}\left\{\left(\frac{1}{\hat{u}_{\hat{\varepsilon}}}-\frac{1}{w_{\hat{\varepsilon}}}\right)+\frac{1}{8}\left(\frac{\zeta_{4}}{\hat{u}_{\hat{\varepsilon}}}-\frac{\zeta_{4}^{\prime}}{w_{\hat{\varepsilon}}}\right)-\frac{5}{24}\left(\frac{\zeta_{3}^{2}}{\hat{u}_{\hat{\varepsilon}}}-\frac{\zeta_{3}^{\prime 2}}{w_{\hat{\varepsilon}}}\right)\right. \\
& \left.-\frac{1}{2 \sqrt{G^{(2)}}}\left(\frac{\zeta_{3}}{\hat{u}_{\hat{\varepsilon}}^{2}}-\frac{\zeta_{3}^{\prime}}{w_{\hat{\varepsilon}}^{2}}\right)-\frac{1}{G^{(2)}}\left(\frac{1}{\hat{u}_{\hat{\varepsilon}}^{3}}-\frac{1}{\hat{w}_{\hat{\varepsilon}}^{3}}\right)\right\}
\end{aligned}
$$

where

$$
\delta_{\hat{\varepsilon}}=1+\frac{1}{8} \zeta_{4}^{\prime}-\frac{5}{24} \zeta_{3}^{\prime 2} .
$$

At the mean, it can be shown that (17) reduces to 


$$
\operatorname{Pr}(X \geq E(X)) \approx 1-\Gamma\left(G^{(1)}(0)\right)+T_{1} T_{2}
$$

where

$$
T_{1}=\frac{\sqrt{G^{(2)}(0)} \gamma\left(G^{(1)}(0)\right)}{1+\frac{1}{8} \zeta_{4}^{\prime}(0)-\frac{5}{24} \zeta_{3}^{\prime 2}(0)}
$$

and

$$
\begin{aligned}
T_{2}= & \frac{1}{6}\left[\zeta_{3}^{\prime}(0)-\zeta_{3}(0)\right]+\frac{1}{40}\left[\zeta_{5}^{\prime}(0)-\zeta_{5}(0)\right] \\
& +\frac{5}{48}\left[\zeta_{3}^{\prime}(0) \zeta_{4}^{\prime}(0)-\zeta_{3}(0) \zeta_{4}(0)\right]+\frac{35}{432}\left[\zeta_{3}^{2}(0)-\zeta_{3}^{\prime 2}(0)\right]
\end{aligned}
$$

\section{THE ANDERSON-DARLING TEST STATISTIC}

\subsection{Background}

The Anderson-Darling (A-D) test is widely used for testing the hypothesis that a sample of size $n$ has been drawn from a population with a specified continuous cumulative distribution function. Anderson and Darling (1952) based their test on the discrepancies between the empirical cumulative distribution function $F_{n}(x)$ and the null cumulative distribution function $F(x)$.

The A-D test statistic is:

$$
A_{n}^{2}=n \int_{-\infty}^{+\infty}\left[F_{n}(x)-F(x)\right]^{2} \psi[F(x)] d F(x)
$$

where $F_{n}(x)$ is the empirical distribution function based on $n$ observations; $\psi(t)(\geq 0)$ is some preassigned weight function. When the weight function is $\psi(t)=1 /[t(1-t)]$, the statistic is:

$$
A_{n}^{2}=n \int_{-\infty}^{+\infty}\left[F_{n}(x)-F(x)\right]^{2} /[F(x)\{1-F(x)\}] d F(x)
$$


The test can be applied from the order statistics, $x_{1} \leq x_{2} \leq \cdots \leq x_{n}$ :

$$
A_{n}^{2}=-n-\frac{1}{n} \sum_{j=1}^{n}(2 j-1)\left[\ln \left(u_{j}\right)+\ln \left(1-u_{n-j+1}\right)\right] \text {, }
$$

where $u_{i}=F\left(x_{i}\right)$. The asymptotic test statistic is

$$
A^{2}=\operatorname{Lim}_{n \rightarrow \infty}\left(A_{n}^{2}\right)
$$

The distribution function of the A-D statistic is complicated, even asymptotically. Consequently, Anderson and Darling (1954) used a numerical method previously applied by Birnbaum (1952) to the Kolmogorov-Smirnov test, to obtain the asymptotic critical points for significance levels of $1 \%, 5 \%$ and $10 \%$ for the A-D test. Lewis (1961) found that the support of the A-D test statistic is essentially from 0 to 8 . He tabulated the distribution function of the A-D test statistic for the asymptotic case (using HermiteGauss numerical quadrature) and for $n=2$ to 8 (using Monte Carlo simulation). When $n$ $=1$ the expression for the c.d.f. requires no integration. For $n>8$, the Monte Carlo method was impractical given the computational constraints at that time. Recently, Marsaglia and Marsaglia (2004) have provided a computational method to evaluate the c.d.f. accurately for any sample size.

Despite the complexity of the c.d.f. for the A-D statistic, the characteristic function is simple and corresponds to that of an infinite weighted sum of independent chi-squared random variables. Exploiting this, Sinclair and Spurr (1988) used the results of Zolotarev (1961) to derive a theoretical function of the upper tail area for the A-D test. The approximations based on this formula are very good above the median. However, the 
approximation is poor in the lower tail, and generates areas in excess of unity in some cases. Sinclair and Spurr derived the first four cumulants of the A-D statistic. Based on its skewness (5.5865) and kurtosis (12.036), they concluded that its distribution should lie between the gamma and log-normal distributions.

\subsection{Saddlepoint Approximations}

Giles (2001) derived a saddlepoint approximation to the asymptotic c.d.f. of the A-D statistic by using the Lugannani and Rice (1980) formula. His numerical results showed that the approximations are excellent (compared with Lewis's “exact” results) in both tails. Given the form of the characteristic function, and the fact that the limit distribution of the A-D statistic is non-normal, Giles suggested that a chi-square base, not a normal base, might be considered.

The characteristic function of $A^{2}$ is

$$
\begin{aligned}
\phi(t) & =\prod_{j=1}^{\infty}[1-2 i t /(j(j+1))]^{-1 / 2} \\
& =\prod_{j=1}^{\infty}\left[1-2 \lambda_{j} i t\right]^{-1 / 2}
\end{aligned}
$$

where $\lambda_{j}=1 /(j(j+1))$ and $i^{2}=-1$. So, the cumulant generating function (c.g.f) of $A^{2}$ is:

$$
K(t)=\log [\theta(t)]=-0.5 \sum_{j=1}^{\infty} \log \left[1-2 \lambda_{j} t\right]
$$

and its derivatives are 


$$
K^{(i)}(t)=d_{i} \sum_{j=1}^{\infty}\left[\lambda_{j} /\left(1-2 \lambda_{j} t\right)\right]^{i} ; d_{1}=1 ; d_{i}=2(i-1) d_{i-1} ; i=2,3,4, \ldots \ldots
$$

Equations (1) - (4) then provide the basic L-R saddlepoint approximation to the c.d.f. of $A^{2}$. When $A^{2}=E\left(A^{2}\right)=K^{(1)}(0)$, equation (5) is used. For any $y$, the saddlepoint equation is:

$$
\sum_{j=1}^{\infty}\left[\lambda_{j} /\left(1-2 \lambda_{j} t\right)\right]-y=0
$$

The solution, $\hat{t}$, is well defined, as $\operatorname{Lim}_{j \rightarrow \infty}\left[\lambda_{j} /\left(1-2 \lambda_{j} t\right)\right]=0$, and is unique. The saddlepoint equation (21) is easily and rapidly solved numerically, for example, by the Newton-Raphson algorithm (which we use), or by a line-search.

Turning to a non-normal-based saddlepoint approximation for the c.d.f. of the A-D statistic, we can apply equations (6) - (10), and equation (11) at the mean. Specifically, we choose a chi-squared base distribution, $\Gamma$. Its c.g.f. (for $\alpha$ degrees of freedom) is:

$$
G\left(w_{\hat{\varepsilon}}\right)=-\frac{\alpha}{2} \log \left(1-2 w_{\hat{\varepsilon}}\right),
$$

whose derivatives are

$$
G^{(i)}\left(w_{\hat{\varepsilon}}\right)=d^{\prime}{ }_{i} \alpha /\left(1-2 w_{\hat{\varepsilon}}\right)^{i} ; d^{\prime}{ }_{1}=1 ; d^{\prime}{ }_{i}=2(i-1) d^{\prime}{ }_{i-1} ; i=2,3,4, \ldots \ldots
$$

To obtain the approximation we first solve for the root $\hat{t}$, using Giles's approximation. Equations (7) and (22) are solved for $w_{\hat{\varepsilon}}$ in terms of $\hat{\varepsilon}$ :

$$
\alpha /\left(1-2 w_{\hat{\varepsilon}}\right)=\hat{\varepsilon},
$$

so that 


$$
w_{\widehat{\varepsilon}}=\frac{1}{2}-\frac{\alpha}{2 \hat{\varepsilon}}
$$

Then we substitute (23) into (6) to solve for the root $\hat{\varepsilon}$ :

$$
\frac{\alpha}{2} \log \left(\frac{\hat{\varepsilon}}{\alpha}\right)-\frac{\hat{\varepsilon}}{2}+\frac{\alpha}{2}=-0.5 \sum_{j=1}^{\infty} \log \left[1-2 \lambda_{j} \hat{t}\right]-y \hat{t}
$$

and substitute $\hat{\varepsilon}$ back into (23) to get $w_{\hat{\varepsilon}}$.

After solving for $\hat{t}, \hat{\varepsilon}$ and $w_{\hat{\varepsilon}}$, we can get $\hat{u}_{\hat{\varepsilon}}$ based on (3) and (10) and evaluate (8). We follow Wood et al.'s suggestion as to the choice of $\alpha$. Matching the derivatives of $K(\hat{t})$ and $G\left(w_{\hat{\varepsilon}}\right)$ implies

$$
\frac{\left[K^{(2)}(\hat{t})\right]^{3}}{\left[K^{(3)}(\hat{t})\right]^{2}}=\frac{\left[G^{(2)}\left(w_{\hat{\varepsilon}}\right)\right]^{3}}{\left[G^{(3)}\left(w_{\hat{\varepsilon}}\right)\right]^{2}}
$$

so that

$$
\alpha=8 \frac{\left[K^{(2)}(\hat{t})\right]^{3}}{\left[K^{(3)}(\hat{t})\right]^{2}} .
$$

Evaluating (25) at $\hat{t}=0$ and $w_{\hat{\varepsilon}}=0$, (26) reduces to

$$
\alpha=2 \frac{\left[K^{(1)}(0)\right]^{2}}{\left[K^{(2)}(0)\right]} .
$$

We also consider $\alpha=2$, because the support for the associated chi-squared distribution is close to that of the A-D test statistic. Other choices of $\alpha$ could be considered. The higher-order approximations are implemented directly, using the results of section 2. 


\section{NUMERICAL EVALUATIONS}

We provide some numerical results to compare the accuracies of the various saddlepoint approximations discussed in section 3. The associated programs were written in double precision FORTRAN, use the Lahey (1992) compiler, and incorporate routines from Press et al. (1992). Table 1 compares the saddlepoint approximations with Lewis's full set of 'exact' results. Chi-Squared (1) (2) (3) denote the chi-squared base with $\alpha$ defined by equations (26) and (27), and $\alpha=2$ respectively.

Table 1 illustrates that Giles's approximation is excellent not only in the tails but also over the whole support of the distribution. The maximum absolute difference between Lewis's and the normal-based approximation is 0.0169 , when the value for the A-D statistic is 0.75 or 0.8 . The chi-squared-based saddlepoint approximations are strictly better than the normal-based saddlepoint approximation in the middle part of the distribution. However, the normal-based approximation performs better in the tail areas, especially in the important right tail area.

Each higher-order approximation performs better (in terms of absolute error) than its loworder counterpart in the middle part of the support (except at the mean of the distribution), and for the first chi-squared base, the higher-order approximation is superior over almost the whole range. However for the other cases, although the higherorder approximations perform better in the middle part of the distribution (except at the mean), they are strictly worse in the right tail. Overall, in the case of the A-D test statistic, although higher-order saddlepoint approximations with either a normal or non- 
normal base distribution can out-perform Giles’s standard Luannani-Rice approximation over much of the support, in the crucial right tail the latter approximation is preferred.

\section{CONCLUDING REMARKS}

Wood et al. (1993) introduced the use of non-normal base distributions for saddlepoint approximations of the type proposed by Lugannani and Rice (1980). Regardless of the base distribution, higher-order approximations of the type considered in this paper are worthy of consideration. Interestingly, this extension appears not to have been discussed or applied previously in the case of a non-normal-based saddlepoint approximation. Using a higher-order saddlepoint approximation in any context does not necessarily guarantee an improvement for any particular fixed finite sample size - it is possible for the corrections from the extra terms to worsen the approximation. The improvement from the extra terms is guaranteed only beyond a certain sample size, but we do not know the exact value of the sample size that is needed for this to occur in practice, as it will vary according to the characteristics of the problem at hand.

This issue has been explored in the context of the Anderson-Darling goodness-of-fit test. We find that while the use of a chi-square base distribution and/or a higher-order expansion can yield improvements over part of the support of the test statistic's distribution, the standard normal-based approximation discussed by Giles (2001) performs commendably well. Most importantly, though the latter approximation is preferred for the right tail critical regions. While there is good intuition to suggest the use 
of a chi-square base distribution for saddlepoint approximations for this particular test statistic, other choices would be worthy of consideration.

\section{ACKNOWLEDGEMENT}

We are grateful to Nilanjana Roy, Min Tsao, Graham Voss and Aman Ullah for their very helpful comments, and to Andrew Wood for confirming that there is a type-setting error in equation (9) of Wood et al. (1993), and that our formula (11) is correct. 
Table 1: Values of $F\left(A^{2}\right)$

\begin{tabular}{|c|c|c|c|c|c|c|c|c|c|}
\hline \multirow[t]{2}{*}{$A^{2}$} & \multirow[t]{2}{*}{ Lewis } & \multicolumn{2}{|c|}{ Normal } & \multicolumn{2}{|c|}{ Chi-Square (1) } & \multicolumn{2}{|c|}{ Chi-Square (2) } & \multicolumn{2}{|c|}{ Chi-Square (3) } \\
\hline & & & High & & & & & & \\
\hline 100 & 0.0000 & 000 & .0000 & 0.0000 & 0000 & 0.0000 & 0.0000 & 0.0000 & .0000 \\
\hline 125 & 0.0003 & 0.0003 & 0.0003 & 0.0003 & 0.0003 & 0.0003 & 0.0003 & 0.0003 & 0.0003 \\
\hline 150 & 0.0014 & .0014 & 0.0014 & 0.0014 & 0.0014 & 0.0014 & 0.0014 & 0.0014 & 0.0014 \\
\hline 175 & 0.0042 & .0043 & 0.0042 & 0.0043 & 0.0042 & 0.0043 & 0.0042 & 0.0042 & 0.0042 \\
\hline 200 & 0.0096 & 99 & 0.0095 & 0098 & 0.0095 & & 0.0095 & 0.0096 & 0.0094 \\
\hline 225 & 0.0180 & 0.0185 & 0.0178 & 0.0185 & 0.0178 & 0.0184 & 0.0178 & 0.0182 & 0.0177 \\
\hline 250 & 0.0296 & 0.0306 & 0.0293 & 0.0305 & 0.0293 & 0.0304 & 0.0292 & 0.0301 & 0.0290 \\
\hline 275 & 0.0443 & .0459 & 0.0439 & 0.0457 & 0.0438 & 0.0456 & 0.0437 & 0.0453 & 0.0434 \\
\hline 300 & 0.0618 & .0641 & 0.0611 & 0.0639 & 0.0611 & 0.0638 & 0.0609 & 0.0635 & 0.0605 \\
\hline 325 & 0.0817 & .0849 & 0.0807 & 0.0845 & 0.0806 & 0.0844 & 0.0805 & 0.0841 & 0.0799 \\
\hline 0.350 & 0.1036 & 0.1077 & 0.1021 & 0.1072 & 0.1020 & 0.1071 & 0.1019 & 0.1068 & 0.1011 \\
\hline 375 & 0.1269 & 0.1320 & 0.1250 & 0.1314 & 0.1248 & 0.1314 & 0.1247 & 0.1311 & 0.1239 \\
\hline 0.400 & 0.1513 & 0.1576 & 0.1489 & 0.1568 & 0.1487 & 0.1567 & 0.1486 & 0.1565 & 0.1477 \\
\hline 0.425 & 0.1764 & 0.1838 & 0.1736 & 0.1829 & 0.1733 & 0.1829 & 0.1733 & 0.1826 & 0.1722 \\
\hline 450 & 0.2019 & 0.2105 & 0.1987 & 0.2094 & 0.1984 & 0.2094 & 0.1984 & 0.2092 & 0.1972 \\
\hline 475 & 0.2276 & 2374 & 0.2241 & 0.2361 & 0.2236 & 0.2361 & 0.2237 & 0.2359 & 0.2225 \\
\hline 500 & 0.2532 & 2641 & 0.2494 & 0.2626 & 0.2489 & 0.2627 & 0.2491 & 0.2625 & 0.2478 \\
\hline 525 & 0.2786 & 2905 & 0.2747 & 389 & 0.2740 & 0.2889 & 0.2743 & 0.2888 & 0.2730 \\
\hline 550 & 0.3036 & & & & & & & 146 & .2979 \\
\hline 575 & 0.3281 & & 0.3244 & & 0.3234 & & & & $82<0$ \\
\hline 0.600 & 0.3520 & & & & & & & & \\
\hline 625 & 0.3753 & 0.3906 & 0.3723 & & & & & & 0.3705 \\
\hline 675 & 0.4199 & 0.4362 & 0.4180 & 0.4338 & 0.4164 & 0.4339 & 0.4176 & 0.4338 & 0.4162 \\
\hline 700 & 0.4412 & 0.4577 & 0.4400 & 0.4553 & 0.4382 & 0.4554 & 0.4395 & 0.4553 & 0.4162 \\
\hline 750 & 0.4815 & 0.4984 & 0.4818 & 0.4959 & 0.4796 & 0.4959 & 0.4814 & 0.4958 & 0.4800 \\
\hline 800 & 0.5190 & 0.5359 & 0.5208 & 0.5333 & 0.5182 & 0.5333 & 0.5204 & 0.5332 & 0.5190 \\
\hline 0.850 & 5537 & 5703 & 0.5569 & .5677 & 0.5541 & 0.5676 & 0.5565 & 0.5675 & 0.5552 \\
\hline 0.900 & 0.5858 & 6018 & 0.5903 & .5993 & 0.5871 & 0.5991 & 0.5899 & 0.5990 & 0.5886 \\
\hline 0.950 & 0.6154 & 0.6307 & 0.6210 & 0.6283 & 0.6176 & 0.6279 & 0.6206 & 0.6279 & 0.6194 \\
\hline 1.000 & 0.6427 & 0.6571 & 0.8952 & 0.6549 & 0.6455 & 0.6544 & 0.8560 & 0.6544 & 0.7651 \\
\hline 1.050 & 0.6680 & 0.6814 & 0.6743 & 0.6794 & 0.6712 & 0.6787 & 0.6746 & 0.6787 & 0.6735 \\
\hline 100 & 0.6912 & 0.7037 & 0.6982 & 0.7018 & 0.6948 & 0.7011 & 0.6982 & 0.7011 & 0.6972 \\
\hline 1.150 & 0.7127 & & 0.7202 & 0.7225 & 0.7164 & 0.7216 & 0.7199 & 0.7216 & 0.7189 \\
\hline 1.200 & 0.7324 & & 0.7401 & & 0.7362 & 0.7405 & & 0.7405 & 0.7389 \\
\hline 1.250 & 0.7580 & & & & & & & 0.7580 & 0.7572 \\
\hline 1.300 & 0.7677 & & 0.7751 & 0.7752 & 0.7713 & 0.7740 & 0.7749 & 0.7741 & 0.7740 \\
\hline 1.350 & 0.7833 & 7911 & 0.7906 & 0.7901 & 0.7868 & 0.7889 & 0.7903 & 0.7889 & 0.7895 \\
\hline
\end{tabular}


Table 1: Continued

\begin{tabular}{|c|c|c|c|c|c|c|c|c|c|}
\hline \multirow[t]{2}{*}{$A^{2}$} & \multirow[t]{2}{*}{ Lewis } & \multicolumn{2}{|c|}{ Normal } & \multicolumn{2}{|c|}{ Chi-Squared (1) } & \multicolumn{2}{|c|}{ Chi-Squared(2) } & \multicolumn{2}{|c|}{ Chi-Squared(3) } \\
\hline & & Low & High & Low & High & Low & High & Low & High \\
\hline 1.400 & 0.7978 & 0.8048 & 0.8048 & 0.8039 & 0.8011 & 0.8026 & 0.8045 & 0.8027 & 0.8038 \\
\hline 1.450 & 0.8111 & 0.8174 & 0.8179 & 0.8167 & 0.8143 & 0.8153 & 0.8177 & 0.8155 & 0.8169 \\
\hline 1.500 & 0.8235 & 0.8292 & 0.8300 & 0.8286 & 0.8265 & 0.8272 & 0.8298 & 0.8273 & 0.8291 \\
\hline 1.550 & 0.8350 & 0.8401 & 0.8412 & 0.8396 & 0.8378 & 0.8381 & 0.8410 & 0.8383 & 0.8403 \\
\hline 1.600 & 0.8457 & 0.8502 & 0.8515 & 0.8498 & 0.8482 & 0.8483 & 0.8513 & 0.8485 & 0.8507 \\
\hline 1.650 & 0.8556 & 0.8596 & 0.8611 & 0.8593 & 0.8579 & 0.8578 & 0.8609 & 0.8580 & 0.8603 \\
\hline 1.700 & 0.8648 & 0.8683 & 0.8700 & 0.8681 & 0.8670 & 0.8666 & 0.8698 & 0.8668 & 0.8693 \\
\hline 1.750 & 0.8734 & 0.8765 & 0.8783 & 0.8764 & 0.8753 & 0.8749 & 0.8781 & 0.8750 & 0.8776 \\
\hline 1.800 & 0.8814 & 0.8840 & 0.8860 & 0.8840 & 0.8831 & 0.8825 & 0.8858 & 0.8827 & 0.8853 \\
\hline 1.850 & 0.8888 & 0.8911 & 0.8931 & 0.8912 & 0.8904 & 0.8897 & 0.8929 & 0.8899 & 0.8925 \\
\hline 1.900 & 0.8957 & 0.8977 & 0.8997 & 0.8978 & 0.8972 & 0.8964 & 0.8996 & 0.8965 & 0.8992 \\
\hline 1.950 & 0.9021 & 0.9039 & 0.9059 & 0.9041 & 0.9035 & 0.9026 & 0.9058 & 0.9028 & 0.9054 \\
\hline 2.000 & 0.9082 & 0.9097 & 0.9117 & 0.9099 & 0.9094 & 0.9084 & 0.9116 & 0.9086 & 0.9112 \\
\hline 2.050 & 0.9138 & 0.9151 & 0.9171 & 0.9153 & 0.9149 & 0.9139 & 0.9170 & 0.9141 & 0.9166 \\
\hline 2.100 & 0.9190 & 0.9201 & 0.9221 & 0.9204 & 0.9200 & 0.9190 & 0.9220 & 0.9192 & 0.9217 \\
\hline 2.150 & 0.9239 & 0.9249 & 0.9268 & 0.9252 & 0.9248 & 0.9238 & 0.9267 & 0.9240 & 0.9264 \\
\hline 2.200 & 0.9285 & 0.9293 & 0.9312 & 0.9296 & 0.9293 & 0.9283 & 0.9311 & 0.9285 & 0.9308 \\
\hline 2.250 & 0.9328 & 0.9334 & 0.9353 & 0.9338 & 0.9335 & 0.9325 & 0.9352 & 0.9327 & 0.9349 \\
\hline 2.300 & 0.9368 & 0.9373 & 0.9392 & 0.9377 & 0.9374 & 0.9364 & 0.9391 & 0.9366 & 0.9388 \\
\hline 2.350 & 0.9405 & 0.9410 & 0.9428 & 0.9413 & 0.9411 & 0.9401 & 0.9427 & 0.9403 & 0.9424 \\
\hline 2.400 & 0.9441 & 0.9444 & 0.9462 & 0.9448 & 0.9446 & 0.9436 & 0.9461 & 0.9438 & 0.9458 \\
\hline 2.450 & 0.9474 & 0.9476 & 0.9493 & 0.9480 & 0.9478 & 0.9468 & 0.9493 & 0.9470 & 0.9490 \\
\hline 2.500 & 0.9504 & 0.9506 & 0.9523 & 0.9510 & 0.9509 & 0.9499 & 0.9522 & 0.9501 & 0.9520 \\
\hline 2.550 & 0.9534 & 0.9535 & 0.9551 & 0.9539 & 0.9537 & 0.9528 & 0.9550 & 0.9529 & 0.9548 \\
\hline 2.600 & 0.9561 & 0.9561 & 0.9577 & 0.9565 & 0.9564 & 0.9555 & 0.9576 & 0.9556 & 0.9574 \\
\hline 2.650 & 0.9586 & 0.9586 & 0.9601 & 0.9590 & 0.9589 & 0.9580 & 0.9601 & 0.9582 & 0.9599 \\
\hline 2.700 & 0.9610 & 0.9610 & 0.9624 & 0.9614 & 0.9613 & 0.9604 & 0.9624 & 0.9606 & 0.9622 \\
\hline 2.750 & 0.9633 & 0.9632 & 0.9646 & 0.9636 & 0.9635 & 0.9627 & 0.9645 & 0.9628 & 0.9644 \\
\hline 2.800 & 0.9654 & 0.9653 & 0.9666 & 0.9657 & 0.9656 & 0.9648 & 0.9666 & 0.9649 & 0.9664 \\
\hline 2.850 & 0.9674 & 0.9673 & 0.9685 & 0.9676 & 0.9676 & 0.9668 & 0.9685 & 0.9669 & 0.9683 \\
\hline 2.900 & 0.9692 & 0.9691 & 0.9703 & 0.9695 & 0.9694 & 0.9686 & 0.9703 & 0.9688 & 0.9701 \\
\hline 2.950 & 0.9710 & 0.9708 & 0.9720 & 0.9712 & 0.9711 & 0.9704 & 0.9720 & 0.9705 & 0.9718 \\
\hline 3.000 & 0.9726 & 0.9725 & 0.9736 & 0.9728 & 0.9728 & 0.9720 & 0.9736 & 0.9722 & 0.9734 \\
\hline 3.050 & 0.9742 & 0.9740 & 0.9751 & 0.9744 & 0.9743 & 0.9736 & 0.9751 & 0.9737 & 0.9749 \\
\hline 3.100 & 0.9756 & 0.9755 & 0.9765 & 0.9758 & 0.9758 & 0.9751 & 0.9765 & 0.9752 & 0.9764 \\
\hline 3.150 & 0.9770 & 0.9768 & 0.9778 & 0.9772 & 0.9771 & 0.9765 & 0.9778 & 0.9766 & 0.9777 \\
\hline 3.200 & 0.9783 & 0.9781 & 0.9791 & 0.9784 & 0.9784 & 0.9778 & 0.9790 & 0.9779 & 0.9789 \\
\hline 3.250 & 0.9795 & 0.9793 & 0.9803 & 0.9796 & 0.9796 & 0.9790 & 0.9802 & 0.9791 & 0.9801 \\
\hline
\end{tabular}


Table 1: Continued

\begin{tabular}{|c|c|c|c|c|c|c|c|c|c|}
\hline \multirow[t]{2}{*}{$A^{2}$} & \multirow[t]{2}{*}{ _ewis } & \multirow{2}{*}{$\begin{array}{l}\text { Norma } \\
\text { Low }\end{array}$} & \multirow{2}{*}{ High } & \multicolumn{2}{|c|}{ Chi-Squared (1) } & \multicolumn{2}{|c|}{ Chi-Squared(2) } & \multicolumn{2}{|c|}{ Chi-Squared(3) } \\
\hline & & & & & & & & & \\
\hline 3.300 & 0.9807 & 9805 & 0.9814 & 0.9808 & 0.9808 & 0.9802 & 0.9813 & 0.9803 & 0.9812 \\
\hline 3.350 & 0.9818 & 0.9816 & 0.9824 & 0.9819 & 0.9818 & 0.9813 & 0.9824 & 0.9814 & 0.9823 \\
\hline 3.400 & 0.9828 & 0.9826 & 0.9834 & 0.9829 & 0.9828 & 0.9823 & 0.9834 & .9824 & 0.9833 \\
\hline 3.450 & 0.9837 & .9835 & 0.9843 & 0.9838 & 0.9838 & .9833 & 0.9843 & .9834 & 0.9842 \\
\hline 3.500 & 0.9846 & 0.9844 & 0.9852 & 0.9847 & 0.9847 & 0.9842 & 0.9852 & 0.9843 & 0.9851 \\
\hline 3.550 & 0.9855 & 0.9853 & 0.9860 & 0.9856 & 0.9855 & 0.9851 & 0.9860 & 0.9852 & 0.9859 \\
\hline 600 & 0.9863 & 0.9861 & 0.9868 & 0.9864 & 0.9863 & 0.9859 & 0.9867 & 0.9860 & 0.9867 \\
\hline 3.650 & 0.9870 & 0.9869 & 0.9875 & 0.9871 & 0.9871 & 0.9867 & & 0.9867 & 0.9874 \\
\hline 3.700 & 0.9878 & 0.9876 & 0.9882 & 0.9878 & 0.9878 & 00974 & & & 0.9881 \\
\hline 3.750 & 0.9884 & 0.9883 & 0000 & & & & & & 9888 \\
\hline 3.800 & 0.9891 & & 0005 & & & & & & 0904 \\
\hline 3.850 & 0.9897 & 0.9895 & 0.9900 & 0.9897 & & & & & 0900 \\
\hline 3.900 & 0.9902 & 0.9901 & 0.9906 & 0.9903 & 0.9903 & 0.9899 & & & 0.9905 \\
\hline 3.950 & 0.9908 & 0.9906 & 0.9911 & 0.9908 & 0.9908 & 0.9905 & 0.9911 & 0.9905 & 0.9910 \\
\hline 4.000 & 0.9913 & 0.9911 & 0.9916 & 0.9913 & 0.9913 & .9910 & & & 0.9915 \\
\hline 4.050 & 0.9917 & 0.9916 & 0.9920 & 0.9918 & 0.9918 & 0.9915 & 0.9920 & & 0.9920 \\
\hline 4.100 & 0.9922 & 0.9921 & 0.9925 & 0.9922 & 0.9922 & 0.9919 & 0.9925 & 0.9920 & 0.9924 \\
\hline 4.150 & 0.9926 & 9925 & 0.9929 & 9926 & 0.9926 & 9924 & 0.9929 & 24 & 0.9928 \\
\hline 4.200 & 0.9930 & 929 & 9933 & & & & & & 9932 \\
\hline 4.250 & 9934 & & & 9934 & & & & & 9936 \\
\hline 4.300 & 0.9938 & & & & & & & & 0.9939 \\
\hline 4.350 & 0.9941 & & & & & & & & \\
\hline 4.400 & 0.9944 & & & & & & & & \\
\hline 4.500 & 0.9950 & & & & & & & & \\
\hline 4.600 & 0.9955 & 0.9954 & 0.9957 & 0.9955 & 0.9955 & 0.9954 & 0.9957 & & 0.9957 \\
\hline 4.700 & 0.9960 & 0.9959 & 0.9961 & 0.9960 & 0.9960 & 0.9958 & 0.9961 & 0.9959 & 0.9961 \\
\hline 4.800 & 0.9964 & 0.9963 & 0.9965 & 0.9964 & 0.9964 & 0.9963 & 0.9965 & 0.9963 & 0.9965 \\
\hline 4.900 & 0.9968 & 9967 & 0.9969 & 0.9968 & 0.9968 & 0.9967 & 0.9969 & 0.9967 & 0.9969 \\
\hline 5.000 & 0.9971 & 970 & 972 & 971 & & & & 0.9970 & 0.9972 \\
\hline 5.500 & 0.9983 & 983 & 9984 & 0.9983 & 983 & 0.9983 & 0.9984 & 0.9983 & 0.9984 \\
\hline 6.000 & 0.9990 & 0.9990 & 0.9991 & 0.9990 & 0.9990 & 0.9990 & 0.9991 & 0.9990 & 0.9991 \\
\hline 7.000 & 0.9997 & 0.9997 & 0.9997 & 0.9997 & 0.9997 & 0.9997 & 0.9997 & 0.9997 & 0.9997 \\
\hline 8.000 & 0.9999 & .9999 & 0.9999 & 9999 & 0.9999 & 0.9999 & 0.9999 & 0.9999 & 0.999 \\
\hline
\end{tabular}

Note: "Low" represent the lower-order saddlepoint approximation; "High" represent the higher-order saddlepoint approximation. 


\section{APPENDIX: PROOF OF HIGHER-ORDER WBB SADDLEPOINT APPROXIMATION}

From the inversion formula for the tail probability:

$$
P(X \geq y)=\frac{1}{2 \pi i} \int_{\hat{\varepsilon}-i \infty}^{\hat{\varepsilon}+i \infty} e^{K(t)-t y} \frac{d t}{t},
$$

and let

$$
\begin{gathered}
G\left(w_{\varepsilon}\right)-\hat{\varepsilon} w_{\varepsilon}=K(t)-t y \\
P(X \geq y)=\frac{1}{2 \pi i} \int_{\hat{\varepsilon}-i \infty}^{\hat{\varepsilon}+i \infty} e^{G\left(w_{\varepsilon}\right)-\hat{\varepsilon} w_{\varepsilon}}\left(\frac{d t}{t d w_{\varepsilon}}\right) d w_{\varepsilon} \\
=\frac{1}{2 \pi i} \int_{\hat{\varepsilon}-i \infty}^{\hat{\varepsilon}+i \infty} e^{G\left(w_{\varepsilon}\right)-\varepsilon w_{\varepsilon}}\left(\frac{1}{w_{\varepsilon}}+\frac{d t}{t d w_{\varepsilon}}-\frac{1}{w_{\varepsilon}}\right) d w_{\varepsilon} \\
=\frac{1}{2 \pi i} \int_{\hat{\varepsilon}-i \infty}^{\hat{\varepsilon}+i \infty} e^{G\left(w_{\varepsilon}\right)-\varepsilon w_{\varepsilon}} \frac{1}{w_{\varepsilon}} d w_{\varepsilon}+\frac{1}{2 \pi i} \int_{\hat{\varepsilon}-i \infty}^{\hat{\varepsilon}+i \infty} e^{G\left(w_{\varepsilon}\right)-\varepsilon w_{\varepsilon}}\left(\frac{d t}{t d w_{\varepsilon}}-\frac{1}{w_{\varepsilon}}\right) d w_{\varepsilon}
\end{gathered}
$$

Again, applying the inversion formula, the first part of (A3) is:

$$
\frac{1}{2 \pi i} \int_{\hat{\varepsilon}-i \infty}^{\hat{\varepsilon}+i \infty} e^{G\left(w_{\varepsilon}\right)-\hat{\varepsilon} w_{\varepsilon}} \frac{1}{w_{\varepsilon}} d w_{\varepsilon}=1-\Gamma(\hat{\varepsilon})
$$

then the second part of (A3) can be written as:

$$
\begin{aligned}
& \frac{1}{2 \pi i} \int_{\hat{\varepsilon}-i \infty}^{\hat{\varepsilon}+i \infty} e^{G\left(w_{\varepsilon}\right)-\varepsilon w_{\varepsilon}}\left(\frac{d t}{t d w_{\varepsilon}}-\frac{1}{w_{\varepsilon}}\right) d w_{\varepsilon} \\
& =\frac{1}{2 \pi i} \int_{\hat{\varepsilon}-i \infty}^{\hat{\varepsilon}+i \infty} e^{G\left(w_{\varepsilon}\right)-\varepsilon w_{\varepsilon}}\left(\frac{d t}{t d w_{\varepsilon}}\right) d w_{\varepsilon}-\frac{1}{2 \pi i} \int_{\hat{\varepsilon}-i \infty}^{\hat{\varepsilon}+i \infty} e^{G\left(w_{\varepsilon}\right)-\varepsilon w_{\varepsilon}} \frac{d w_{\varepsilon}}{w_{\varepsilon}} .
\end{aligned}
$$

Let

$$
I_{1}=\frac{1}{2 \pi i} \int_{\hat{\varepsilon}-i \infty}^{\hat{\varepsilon}+i \infty} e^{G\left(w_{\varepsilon}\right)-\delta w_{\varepsilon}}\left(\frac{d t}{t d w_{\varepsilon}}\right) d w_{\varepsilon}
$$




$$
I_{2}=\frac{1}{2 \pi i} \int_{\hat{\varepsilon}-i \infty}^{\hat{\varepsilon}+i \infty} e^{G\left(w_{\varepsilon}\right)-\delta w_{\varepsilon}} \frac{d w_{\varepsilon}}{w_{\varepsilon}}
$$

from (A2):

$$
I_{1}=\frac{1}{2 \pi i} \int_{\hat{\varepsilon}-i \infty}^{\hat{\varepsilon}+i \infty} e^{K(t)-t y} \frac{d t}{t} .
$$

Using Daniels’s (1987) result (3.3) we get:

$$
\begin{aligned}
& I_{1}=\frac{e^{K-\hat{t} y}}{\hat{t} \sqrt{2 \pi K^{(2)}}}\left(1+\frac{1}{8} \zeta_{4}-\frac{5}{24} \zeta_{3}^{2}-\frac{\zeta_{3}}{2 \hat{t}\left(K^{(2)}\right)^{1 / 2}}-\frac{1}{\hat{t}^{2} K^{(2)}}\right) \\
& I_{2}=\frac{e^{G\left(w_{\hat{\varepsilon}}\right)-\hat{\varepsilon} w_{\hat{\varepsilon}}}}{w_{\hat{\varepsilon}} \sqrt{2 \pi G^{(2)}}}\left(1+\frac{1}{8} \zeta_{4}^{\prime}-\frac{5}{24} \zeta_{3}^{\prime 2}-\frac{\zeta_{3}^{\prime}}{2 w_{\hat{\varepsilon}}\left(G^{(2)}\right)^{1 / 2}}-\frac{1}{w_{\hat{\varepsilon}}^{2} G^{(2)}}\right)
\end{aligned}
$$

Daniels's saddlepoint approximation to the density function at any specified point $y$ up to $b_{1}$ term is:

$$
f(y)=g(y)\left\{1+\frac{1}{8} \zeta_{4}(\hat{t})-\frac{5}{24} \zeta_{3}^{2}(\hat{t})+\ldots\right\} .
$$

Let

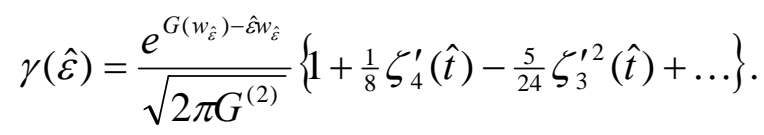

Now we can rewrite $I_{1}$ and $I_{2}$ as:

$$
\begin{aligned}
& I_{1}=\frac{e^{G\left(w_{\hat{\varepsilon}}\right)-\hat{\varepsilon} w_{\hat{\varepsilon}}}}{\sqrt{2 \pi G^{(2)}}} \frac{\left\{1+\frac{1}{8} \zeta_{4}^{\prime}-\frac{5}{24} \zeta_{3}^{\prime 2}\right\}}{\left\{1+\frac{1}{8} \zeta_{4}^{\prime}-\frac{5}{24} \zeta_{3}^{\prime 2}\right\}} \frac{\sqrt{2 \pi G^{(2)}}}{\hat{t} \sqrt{2 \pi K^{(2)}}}\left(1+\frac{1}{8} \zeta_{4}-\frac{5}{24} \zeta_{3}^{2}-\frac{\zeta_{3}}{2 \hat{t}\left(K^{(2)}\right)^{1 / 2}}-\frac{1}{\hat{t}^{2} K^{(2)}}\right) \\
& =\gamma(\hat{\varepsilon}) \frac{\sqrt{G^{(2)}}}{\hat{t} \sqrt{K^{(2)}}\left\{1+\frac{1}{8} \zeta_{4}^{\prime}-\frac{5}{24} \zeta_{3}^{\prime 2}\right\}}\left(1+\frac{1}{8} \zeta_{4}-\frac{5}{24} \zeta_{3}^{2}-\frac{\zeta_{3}}{2 \hat{t}\left(K^{(2)}\right)^{1 / 2}}-\frac{1}{\hat{t}^{2} K^{(2)}}\right) \\
& I_{2}=\frac{e^{G\left(w_{\hat{\varepsilon}}\right)-\hat{\varepsilon} w_{\hat{\varepsilon}}}}{\sqrt{2 \pi G^{(2)}}} \frac{\left\{1+\frac{1}{8} \zeta_{4}^{\prime}-\frac{5}{24} \zeta_{3}^{\prime 2}\right\}}{w_{\hat{\varepsilon}}\left\{1+\frac{1}{8} \zeta_{4}^{\prime}-\frac{5}{24} \zeta_{3}^{\prime 2}\right\}}\left(1+\frac{1}{8} \zeta_{4}^{\prime}-\frac{5}{24} \zeta_{3}^{\prime 2}-\frac{\zeta_{3}^{\prime}}{2 w_{\hat{\varepsilon}}\left(G^{(2)}\right)^{1 / 2}}-\frac{1}{w_{\hat{\varepsilon}}^{2} G^{(2)}}\right) \\
& =\gamma(\hat{\varepsilon}) \frac{1}{w_{\hat{\varepsilon}}\left\{1+\frac{1}{8} \zeta_{4}^{\prime}-\frac{5}{24} \zeta_{3}^{\prime 2}\right\}}\left(1+\frac{1}{8} \zeta_{4}^{\prime}-\frac{5}{24} \zeta_{3}^{\prime 2}-\frac{\zeta_{3}^{\prime}}{2 w_{\hat{\varepsilon}}\left(G^{(2)}\right)^{1 / 2}}-\frac{1}{w_{\hat{\varepsilon}}^{2} G^{(2)}}\right) .
\end{aligned}
$$


Let

$$
\begin{aligned}
& \hat{u}_{\hat{\varepsilon}}=\hat{t} \sqrt{K^{(2)}}\left[G^{(2)}\right]^{-1 / 2} \\
& \delta_{\hat{\varepsilon}}=1+\frac{1}{8} \lambda_{4}^{\prime}-\frac{5}{24} \lambda_{3}^{\prime 2} .
\end{aligned}
$$

Then,

$$
\begin{aligned}
P(X \geq y)=1 & -\Gamma(\hat{\varepsilon})+I_{1}-I_{2} \\
\approx & 1-\Gamma(\hat{\varepsilon})+\gamma(\hat{\varepsilon}) \frac{1}{\hat{u}_{\hat{\varepsilon}} \delta_{\hat{\varepsilon}}}\left(1+\frac{1}{8} \lambda_{4}-\frac{5}{24} \lambda_{3}^{2}-\frac{\lambda_{3}}{2 \hat{t}\left(K^{(2)}\right)^{1 / 2}}-\frac{1}{\hat{t}^{2} K^{(2)}}\right) \\
& -\gamma(\hat{\varepsilon}) \frac{1}{\hat{w} \delta_{\hat{\varepsilon}}}\left(1+\frac{1}{8} \lambda_{4}^{\prime}-\frac{5}{24} \lambda_{3}^{\prime 2}-\frac{\lambda_{3}^{\prime}}{2 \hat{w}\left(G^{(2)}\right)^{1 / 2}}-\frac{1}{\hat{w}^{2} G^{(2)}}\right) \\
= & 1-\Gamma(\hat{\varepsilon})+\frac{\gamma(\hat{\varepsilon})}{\delta_{\hat{\varepsilon}}}\left\{\left(\frac{1}{\hat{u}_{\hat{\varepsilon}}}-\frac{1}{w_{\hat{\varepsilon}}}\right)+\frac{1}{8}\left(\frac{\lambda_{4}}{\hat{u}_{\hat{\varepsilon}}}-\frac{\lambda_{4}^{\prime}}{w_{\hat{\varepsilon}}}\right)-\frac{5}{24}\left(\frac{\lambda_{3}^{2}}{\hat{u}_{\hat{\varepsilon}}}-\frac{\lambda_{3}^{\prime 2}}{w_{\hat{\varepsilon}}}\right)\right. \\
- & \left.\frac{1}{2 \sqrt{G^{(2)}}}\left(\frac{\lambda_{3}}{\hat{u}_{\hat{\varepsilon}}^{2}}-\frac{\lambda_{3}^{\prime}}{w_{\hat{\varepsilon}}^{2}}\right)-\frac{1}{G^{(2)}}\left(\frac{1}{\hat{u}_{\hat{\varepsilon}}^{3}}-\frac{1}{\hat{w}_{\hat{\varepsilon}}^{3}}\right)\right\}
\end{aligned}
$$

When $X=E(X), w_{\hat{\varepsilon}}=0$ and $\hat{u}_{\varepsilon}=0$. Therefore, we need to take the limit of (A6).

First, (A6) can be written as:

$$
\operatorname{Pr}(X \geq y)=S_{1}+\frac{\gamma(\hat{\varepsilon})}{\delta_{\hat{\varepsilon}}} \sqrt{G^{(2)}}\left(S_{2}-S_{3}+S_{4}\right)
$$

where

$$
\begin{aligned}
& S_{1}=1-\Gamma(\hat{\varepsilon})+\frac{\gamma(\hat{\varepsilon})}{\delta_{\hat{\varepsilon}}}\left(\frac{1}{\hat{u}_{\hat{\varepsilon}}}-\frac{1}{w_{\hat{\varepsilon}}}\right) \\
& S_{2}=\frac{\zeta_{4}}{8 \hat{t} \sqrt{K^{(2)}}}-\frac{5 \zeta_{3}^{2}}{24 \hat{t} \sqrt{K^{(2)}}}-\frac{\zeta_{3}}{2 \hat{t}^{2} K^{(2)}}-\frac{1}{\hat{t}^{3}\left(K^{(2)}\right)^{3 / 2}}+\frac{1}{\left[2\left(\hat{t} K^{(1)}-K(\hat{t})\right]^{3 / 2}\right.} \\
& S_{3}=\frac{\zeta_{4}^{\prime}}{8 w_{\hat{\varepsilon}} \sqrt{G^{(2)}}}-\frac{5 \zeta_{3}^{\prime 2}}{24 w_{\hat{\varepsilon}} \sqrt{G^{(2)}}}-\frac{\zeta_{3}^{\prime}}{2 w_{\hat{\varepsilon}}^{2} G^{(2)}}-\frac{1}{\hat{w}_{\hat{\varepsilon}}^{3}\left(G^{(2)}\right)^{3 / 2}}+\frac{1}{\left[2\left(w_{\hat{\varepsilon}} G^{(1)}-G\left(w_{\hat{\varepsilon}}\right)\right]^{3 / 2}\right.} \\
& S_{4}=\frac{1}{\left[2\left(w_{\hat{\varepsilon}} G^{(1)}-G\left(w_{\hat{\varepsilon}}\right)\right]^{3 / 2}\right.}-\frac{1}{\left[2\left(\hat{t} K^{(1)}-K(\hat{t})\right]^{3 / 2}\right.} .
\end{aligned}
$$


We can see $S_{1}$ is the same as the formula of Wood et al. (1993). Therefore the limit of $S_{1}$ is (8). Comparing the expressions for $S_{2}$ and $S_{3}$ with that for $b_{1}$ in (13), using Daniels's results we can get the limit of $S_{2}$ and $S_{3}$ as:

$$
\begin{aligned}
& \operatorname{Lim}_{\hat{t} \rightarrow 0} S_{2}=\frac{1}{40} \zeta_{5}-\frac{5}{48} \zeta_{3} \zeta_{4}+\frac{35}{432} \zeta_{3}^{2} \\
& \operatorname{Lim}_{i \rightarrow 0} S_{3}=\frac{1}{40} \zeta_{5}^{\prime}-\frac{5}{48} \zeta_{3}^{\prime} \zeta_{4}^{\prime}+\frac{35}{432} \zeta_{3}^{\prime 2} .
\end{aligned}
$$

Then we apply L'Hôpital's rule to get the limit of $S_{4}$ :

$$
\operatorname{Lim}_{\hat{t} \rightarrow 0} S_{4}=\operatorname{Lim}_{\hat{t} \rightarrow 0} \frac{\left[\hat{t} K^{(1)}-K(\hat{t})\right]^{3 / 2}-\left[w_{\hat{\varepsilon}} G^{(1)}-G\left(w_{\hat{\varepsilon}}\right)\right]^{3 / 2}}{2 \sqrt{2}\left[w_{\hat{\varepsilon}} G^{(1)}-G\left(w_{\hat{\varepsilon}}\right)\right]^{3 / 2}\left[\hat{t} K^{(1)}-K(\hat{t})\right]^{3 / 2}} .
$$

Differentiating both denominator and numerator of (A8):

$$
\begin{aligned}
\operatorname{Lim}_{\hat{t} \rightarrow 0} S_{4}=\operatorname{Lim}_{\hat{t} \rightarrow 0} & \frac{1}{2 \sqrt{2}} \frac{\frac{3}{2} \hat{t} K^{(2)} \sqrt{\hat{t} K^{(1)}-K}-\frac{3}{2} \hat{t} \hat{t} K^{(2)} \sqrt{w_{\hat{\varepsilon}} G^{(1)}-G}}{\sqrt{\hat{t} K^{(1)}-K}\left[w_{\hat{\varepsilon}} G^{(1)}-G\right]^{3 / 2}+\frac{3}{2} \hat{t} K^{(2)} \sqrt{w_{\hat{\varepsilon}} G^{(1)}-G}\left[\hat{t} K^{(1)}-K\right]^{3 / 2}} \\
& =\operatorname{Lim}_{\hat{t} \rightarrow 0} \frac{1}{2 \sqrt{2}} \frac{\sqrt{\hat{t} K^{(1)}-K}-\sqrt{w_{\hat{\varepsilon}} G^{(1)}-G}}{\sqrt{\hat{t} K^{(1)}-K}\left[w_{\hat{\varepsilon}} G^{(1)}-G\right]^{3 / 2}+\sqrt{w_{\hat{\varepsilon}} G^{(1)}-G}\left[\hat{t} K^{(1)}-K\right]^{3 / 2}} .
\end{aligned}
$$

Differentiating again and applying L'Hôpital's rule a second time :

$$
\begin{aligned}
& \operatorname{Lim}_{\hat{t} \rightarrow 0} S_{4}=\operatorname{Lim}_{\hat{t} \rightarrow 0} \frac{1}{2 \sqrt{2}} \frac{\sqrt{w_{\hat{\varepsilon}} G^{(1)}-G}-\sqrt{\hat{t} K^{(1)}-K}}{\left(w_{\hat{\varepsilon}} G^{(1)}-G\right)^{2}+6\left(\hat{t} K^{(1)}-K\right)\left(w_{\hat{\varepsilon}} G^{(1)}-G\right)+\left(\hat{t} K^{(1)}-K\right)^{2}} \\
& \operatorname{Lim}_{\hat{t} \rightarrow 0} S_{4}=\operatorname{Lim}_{\hat{t} \rightarrow 0}-\frac{64}{2 \sqrt{2}}\left[\frac{1}{\left(w_{\hat{\varepsilon}} G^{(1)}-G\right)^{3 / 2}}-\frac{1}{\left(\hat{t} K^{(1)}-K\right)^{3 / 2}}\right]=\operatorname{Lim}_{\hat{t} \rightarrow 0}-64 S_{4}
\end{aligned}
$$

so that $\operatorname{Lim}_{\hat{t} \rightarrow 0} S_{4}=0$.

Thus, when $X=E(X)$, we obtain the formula in (18). 


\section{REFERENCES}

Anderson, T. W. and Darling, D. A. (1952), “Asymptotic Theory of Certain "Goodness of Fit”, Criteria Based on Stochastic Processes”, Annals of Mathematical Statistics, 23, 193-212.

Anderson, T. W. and Darling, D. A. (1954), “A Test of Goodness of Fit”, Journal of the American Statistical Association, 49, 765-769.

Barndorff-Nielsen (1991), "Modified Signed Log-Likelihood Ratio”, Biometrika, 78, 557-563.

Brinbaum, Z. W. (1952), “Numerical Tabulation of the Distribution of Kolmogorov's Statistic for Finite Sample Size”, Journal of the American Statistical Association, 47, 425-441.

Daniels, H. E. (1954), “Saddlepoint Approximations in Statistics”, Annals of Mathematical Statistics, 25, 631-650.

Daniels, H. E. (1980), “Exact Saddlepoint Approximations”, Biometrika, 67, 59-63.

Daniels, H. E. (1987), “Tail Probability Approximations”, International Statistical Review, 55, 37-48.

Field, C. and Ronchetti, E. (1990), Small Sample Asymptotics, Institute of Mathematical Satistics.

Giles, D. E. A. (2001), “A Saddlepoint Approximation to the Distribution Function of the Anderson-Darling Test Statistic”, Communications in Statistics, B, 30, 899-905.

Goustis, C. and Casella, G. (1999), "Explaining the Saddlepoint Approximation", American Statistician, 53, 216-224. 
Huzurbazar, S. (1999), "Practical Saddlepoint Approximations”, American Statistician, $53,225-232$.

Lahey Computer Systems (1992), Lahey F77L Fortran Programmer's Reference Manual, Incline Village, NV.: Lahey Computer Systems.

Lewis, P. A. W. (1961), "Distribution of the Anderson-Darling Statistic", Annals of Mathematical Statistics, 32, 1118-1124.

Lugannani, R. and Rice, S. O. (1980), "Saddlepoint Approximation for the Distribution of the Sum of Independent Random Variables”, Advances in Applied Probability, 12, 475-90.

Marsaglia, G. and Marsaglia, J. C. W. (2004) "Evaluating the Anderson-Darling Distribution”, http://www.jstatsoft.org/v09/i02/ad.pdf.

Press, W. H., Teukolsky, S. A., Vetterling, W. T. and Flannery, B. P. (1992), Numerical Recipes in FORTRAN: The Art of Scientific Computing, $2^{\text {nd }}$ ed., Cambridge: Cambridge University Press.

Reid, N. (1988), "Saddlepoint Methods and Statistical Inference”, (with discussion), Statistical Science, 3, 213-238.

Reid, N. (1991), “Approximations and Asymptotics”, in Statistical Theory and Models, Essays in Honor of D. R. Cox, Chapman and Hall, 287-334.

Routledge, R. and Tsao, M. (1997), "On the Relationship Between Two Asymptotic Expansions for the Distribution of Sample Mean and its Applications”, the Annals of Statistics, 25, 2200-2209. 
Sinclair, C. D. and Spurr, B. D. (1988), “Approximations to the Distribution Function of the Anderson-Darling Test Statistic”, Journal of the American Statistical Association, 83, 1190-1191.

Wood, A. T. A., Booth, J. G. and Butler, R. W. (1993) "Saddlepoint Approximations to the CDF of Some Statistics with Nonnormal Limit Distributions”, Journal of the American Statistical Association, 88, 680-686.

Zolotarev, V. M. (1961), “Concerning a Certain Probability Problem”, Theory of Probability and its Applications, 6, 201-204. 